\title{
Basis in the Space of $C^{\infty}$-Functions on a Graduated Sharp Cusp
}

\author{
By A.P. Goncharov and V.P. Zahariuta
}

\begin{abstract}
Using the basis in the space of Whitney functions $\mathcal{E}(K)$, where $K \subset \mathbb{R}$ is the closure of a union of a sequence of closed intervals tending to a point, we construct a special basis in the space $C^{\infty}[0,1]$ and then a basis in the space of $C^{\infty}$-functions on a graduated sharp cusp with arbitrary sharpness.
\end{abstract}

\section{Introduction}

The Grothendieck problem about the existence of bases in a nuclear Fréchet space was solved in the negative by Zobin and Mityagin [21]. Still there is no example of a concrete functional nuclear F-space without a basis. The space of $C^{\infty}$-functions on a sharp cusp has been considered for a long time as a candidate for this role ([2], see also [20]). It should be noted that Domański and Vogt proved recently in [3] that the space of real-analytic functions on the open domain has no basis, but this space is not metrizable.

If a bounded domain $\Omega \in \mathbb{R}^{d}$ has smooth enough boundary, then the space $C^{\infty}(\bar{\Omega})$ has a basis. Mityagin proved ([12], L. 25) that the Chebyshev polynomials form a basis in the space $C^{\infty}[-1,1]$ (see also [8]); the case of domains with smooth boundaries was considered by Triebel [16] and Baouendi-Goulaouic [1]; Zerner [20] did so for domains with Lipschitz boundaries. The existence of a basis in the space $C^{\infty}(\bar{\Omega})$, where $\Omega$ has a boundary of Hölder type was established in [13, 17] (see also [4]). Zeriahi in [19] found a basis in the space of Whitney functions $\mathcal{E}(K)$ for a compact set $K$ satisfying the Markov property.

In all these cases the space $C^{\infty}(\bar{\Omega})$ turns to be isomorphic to the space $s$ of rapidly decreasing sequences and the desired basis is the system of orthogonal polynomials in an appropriate Hilbert space. In the case of domain $\Omega$ with a cusp (more sharp than Hölder type) the spaces $C^{\infty}(\bar{\Omega})$ and $s$ are not isomorphic and moreover a continuum of pairwise non-isomorphic spaces of this kind were found in $[14,5]$ by the method of linear topological invariants.

Applying a modification of Mityagin's basis construction in the space $C^{\infty}(\mathbb{R})([12]$, T. 15), bases were constructed for $C_{F}^{\infty}(\bar{\Omega})$, when $\Omega \in \mathbb{R}^{2}$ is a graduated sharp cusp [9], and for $\mathcal{E}_{F}(K)$, when $K \subset \mathbb{R}$ is the closure of a union of a sequence of closed intervals tending to a point [6]. The subscript $F$ here means flat and corresponds to the subspaces of functions vanishing with all derivatives at the point of the cusp of $\Omega$, (respectively at the point of accumulation of the intervals

Math Subject Classifications. primary 46E10; secondary 46A35. 
of $K$ ). It should be noted that in the classes of spaces $C_{F}^{\infty}(\bar{\Omega}), \mathcal{E}_{F}(K)$ a continuum of pairwise non-isomorphic spaces can be found as well.

New construction of a basis in the whole space $\mathcal{E}(K)$ was suggested in [7] for the compact set $K$ of above-mentioned type. The method works under some restrictions on $K$, but it can be applied in two important cases: first, when the space $\mathcal{E}(K)$ is isomorphic to the space $s$ and, second, when there are severe constrains on the distances between neighboring intervals but the only restriction on the sequence of interval's lengths is its monotonicity. The last case is of especial interest, because it contains a continuum of pairwise non-isomorphic spaces.

In the present article we construct a basis in the space $C^{\infty}(\bar{\Omega}), \Omega$ is a plane domain of the form of graduated sharp cusp with arbitrary sharpness of the spike. In the construction we use a special basis in the space $C^{\infty}[0,1]$ (Section 3 ). The present basis can not be obtained as an expansion of the basis in the subspace $C_{F}^{\infty}(\bar{\Omega})$ since this subspace is not complemented in the space $C^{\infty}(\bar{\Omega})$ (see Remark 6.3 in [7]).

\section{Preliminaries}

Let $\Omega \subset \mathbb{R}^{d}$ be a bounded domain, $K \subset \mathbb{R}^{d}$ be a compact set. We consider the space $C^{\infty}(\bar{\Omega})$ of infinitely differentiable in $\Omega$ functions such that the functions and all their derivatives are uniformly continuous on the domain, and the space $\mathcal{E}(K)$ of Whitney functions, that is traces on $K$ of $C^{\infty}$-functions defined on all space $\mathbb{R}^{d}$. The topology in the space $C^{\infty}(\bar{\Omega})$ is defined by the norms

$$
|f|_{p}=\sup \left\{\left|f^{(j)}(z)\right|: z \in \Omega,|j| \leq p\right\}, p \in \mathbb{N}_{0}:=\{0,1, \ldots\},
$$

where $j=\left(j_{1}, \ldots, j_{d}\right) \in \mathbb{N}_{0}^{d}$ and $|j|=j_{1}+\ldots+j_{d}$.

In turn the norms in the space $\mathcal{E}(K)$ are defined by

$$
\|f\|_{p}=|f|_{p}+\sup \left\{\frac{\left|\left(R_{z_{0}}^{p} f\right)^{(j)}(z)\right|}{\left|z-z_{0}\right|^{p-|j|}}: z, z_{0} \in K, z \neq z_{0},|j| \leq p\right\}
$$

$p \in \mathbb{N}_{0}$, where $R_{z_{0}}^{p} f(z)=f(z)-T_{z_{0}}^{p} f(z)$ is the Taylor remainder. In what follows we will consider only the cases $d=1$ or $d=2$.

The space $\mathcal{E}(K)$ is always nuclear as a quotient space of $s$. If the domain $\Omega$ is regular in Whitney sense (for the definition see e. g., [10]), then the norms $|\cdot|_{p}$ and $\|\cdot\|_{p}$ are equivalent and thus, $C^{\infty}(\bar{\Omega}) \simeq \mathcal{E}(\bar{\Omega})$.

We use the Chebyshev polynomials

$$
T_{n}(x)=\cos (n \cdot \arccos x),|x| \leq 1, n \in \mathbb{N}_{0} .
$$

Let $\tilde{T}_{n}$ be the Chebyshev polynomial considered on $\mathbb{R}$ and for fixed interval $I_{k}=\left[x_{k}-\delta_{k}, x_{k}+\right.$ $\left.\delta_{k}\right] \subset K$ let $\tilde{T}_{n k}$ denote the scaling Chebyshev polynomial, that is $\tilde{T}_{n k}(x)=\tilde{T}_{n}\left(\frac{x-x_{k}}{\delta_{k}}\right)$, and let $T_{n k}$ be the restriction of $\tilde{T}_{n k}$ on $I_{k}, T_{n k}=0$ otherwise on $K$.

By $\xi_{n k}$ we denote the functional $\xi_{n k}(f)=\frac{2}{\pi} \int_{0}^{\pi} f\left(x_{k}+\delta_{k} \cos t\right) \cos n t d t$, $n \in \mathbb{N}_{0}$ (if $n=0$, then we take 1 instead of 2 in the coefficient). Clearly, for fixed $k$ the functionals $\left(\xi_{n k}\right)$ are biorthogonal to the system $\left(\tilde{T}_{n k}\right)$.

By $|\cdot|_{-q}$ we denote the dual norm of a functional in the corresponding space. We adhere to the convention that $0^{0}=1$. 


\section{Special basis in the space $C^{\infty}[0,1]$}

Let us fix a compact set $K=\{0\} \cup \bigcup_{k=1}^{\infty} I_{k} \subset[0,1]$, where $I_{k}=\left[a_{k}, b_{k}\right]=\left[x_{k}-\right.$ $\left.\delta_{k}, x_{k}+\delta_{k}\right]$ be such that $\delta_{k} \downarrow 0$ and for some constant $C$ one has $x_{k} \leq C \delta_{k}, \delta_{k} \leq C \delta_{k+1}$ and for $h_{k}:=\operatorname{dist}\left(I_{k}, I_{k+1}\right)$ let $C h_{k} \geq \delta_{k}, \forall k$. Without loss of generality we can take the compact set $K=\{0\} \cup \bigcup_{k=1}^{\infty}\left[3 \cdot 2^{-k-1}, 2^{-k+1}\right]$ the same as in [7], Section 6. Then the following functions $\left\{e_{n k}\right\}_{n=0, k=1}^{\infty, \infty}$ form a basis in the space $\mathcal{E}(K)$. For $l(k)=[k / 4]$ (the greatest integer in $k / 4$ ) let $e_{n k}=\left.\tilde{T}_{n k}\right|_{\left[0, b_{k}\right] \cap K}$ and $e_{n k}=0$ otherwise on $K$ if $n<l(k) ; e_{n k}=T_{n k}$ if $n \geq l(k)$. The system of functionals $\left\{\eta_{n k}\right\}_{n=0, k=1}^{\infty, \infty}$ with

$$
\eta_{n k}=\xi_{n k}-\sum_{i=n}^{l(k-1)-1} \xi_{n k}\left(e_{i k-1}\right) \xi_{i k-1}, n<l(k) ; \eta_{n k}=\xi_{n k}, n \geq l(k),
$$

is biorthogonal to the system $\left\{e_{n k}\right\}_{n=0, k=1}^{\infty, \infty}$.

In addition for $i \leq n$ we have the following bounds [7]:

$$
\begin{aligned}
\sup \left\{\left|T_{n k}^{(i)}(x)\right|:\left|x-x_{k}\right| \leq \delta_{k}\right\} & \leq n^{2 i} \delta_{k}^{-i}, n \in \mathbb{N}_{0} ; \\
\left|\xi_{n k}\right|_{-q} & \leq C\left(\delta_{k} / n\right)^{\min (n, q)}, n \in \mathbb{N}_{0} ; \\
\sup \left\{\left|\tilde{T}_{n k}^{(i)}(x)\right|: 0 \leq x \leq b_{k}\right\} & \leq \delta_{k}^{-2 i-1}, n<l(k) ; \\
\left|\eta_{n k}\right|_{-q} & \leq C \delta_{k}^{q-1}, n<l(k) .
\end{aligned}
$$

To simplify notation we use the same letter $C$ for any constant which does not depend on $n$ and $k$.

We will extend the basis elements $e_{n k}$ from $K$ onto [0,1]. In this way we obtain a continuous projection in the space $C^{\infty}[0,1]$. This idea goes back to Mityagin's construction of basis in the space $C^{\infty}(\mathbb{R})([12]$, T. 15; see also [9]).

Let $\omega_{\tau}$ be a $C^{\infty}$-function such that $\omega_{\tau}(x)=1$ for $x \leq 0, \omega_{\tau}(x)=0$ for $x \geq \tau$ and $\left|\omega_{\tau}\right|_{p} \leq$ $C_{p} \tau^{-p}, p \in \mathbb{N}_{0}$. Now let $\omega_{0 k}(x)=\omega_{\delta_{k}}\left(x-b_{k}\right)$ and for $\tau=\delta_{k} \cdot n^{-2}$ let $\omega_{n k}(x)=\omega_{\tau}\left(x-b_{k}\right)$ if $0<n<l(k), \omega_{n k}(x)=\omega_{\tau}\left(x-b_{k}\right)\left[1-\omega_{\tau}\left(x-a_{k}+\tau\right)\right]$ if $n \geq l(k)$. Clearly

$$
\left|\omega_{0 k}\right|_{p} \leq C \delta_{k}^{-p},\left|\omega_{n k}\right|_{p} \leq C \delta_{k}^{-p} n^{2 p}, n \in \mathbb{N} .
$$

Define $\tilde{e}_{n k}=\tilde{T}_{n k} \cdot \omega_{n k}$. Fix $F \in C^{\infty}[0,1]$. Let $f=F \mid K$. Using the basis expansion $f=\sum_{k=1}^{\infty} \sum_{n=0}^{\infty} \eta_{n k}(f) \cdot e_{n k}$ we introduce the following linear operator

$$
Q: C^{\infty}[0,1] \rightarrow C^{\infty}[0,1]: F \mapsto \sum_{k=1}^{\infty} \sum_{n=0}^{\infty} \eta_{n k}(f) \cdot \tilde{e}_{n k}
$$

Lemma 3.1. $Q$ is a continuous projection.

Proof. Since $\left.\tilde{e}_{n k}\right|_{K}=e_{n k}$, we see that $\left.Q(F)\right|_{K}=f$ and $Q$ is a projection. Let us show its continuity. Given $p \in \mathbb{N}_{0}$ let $q=2 p+3$. Fix $k>4 q$.

For each polynomial $P$ the Bernstein theorem (see e. g., [15]) implies

$$
|P(x)| \leq\left|x+\sqrt{x^{2}-1}\right|^{\operatorname{deg} P} \sup \{|P(x)|:|x| \leq 1\}, \quad|x|>1 .
$$


For Chebyshev polynomials we get $\left|\tilde{T}_{n}^{(i)}(1+\varepsilon)\right| \leq(1+2 \sqrt{\varepsilon})^{n} T_{n}^{(i)}(1)$ if $\varepsilon \leq 1 / 4$ and $\left|\tilde{T}_{n}^{(i)}(x)\right| \leq$ $e^{2} n^{2 i}$ for $|x| \leq 1+n^{-2}$. It follows that if $\operatorname{dist}\left(x, I_{k}\right) \leq \delta_{k} n^{-2}$ then

$$
\left|\tilde{T}_{n k}^{(i)}(x)\right| \leq e^{2} n^{2 i} \delta_{k}^{-i}
$$

Now we can estimate the norms of $\tilde{e}_{n k}$.

Let at first $0<n<l(k)$. Then $\tilde{e}_{n k}=\tilde{T}_{n k}$ for $x \leq b_{k} ; \tilde{e}_{n k}=0$ for $x \geq b_{k}+\delta_{k} n^{-2}$. For other $x$ using the Leibnitz formula by (3.5) and (3.6) we get $\left|\tilde{e}_{n k}^{(j)}(x)\right| \leq C n^{2 j} \delta_{k}^{-j}, j \in \mathbb{N}_{0}$. Since $n<l(k) \leq k / 4$ and $\delta_{k}=2^{-k-2}$, we see that $n^{2 j} \leq \delta_{k}^{-j}$. Taking into account (3.3), we obtain the bound

$$
\left|\tilde{e}_{n k}\right|_{p} \leq C \delta_{k}^{-2 p-1}
$$

Clearly it is valid also for $n=0$.

Let now $n \geq l(k)$. Then $\tilde{e}_{n k}=T_{n k}$ on $I_{k}$ and $\tilde{e}_{n k}(x)=0$ for $\operatorname{dist}\left(x, I_{k}\right) \geq \delta_{k} n^{-2}$. In the same manner we can see that

$$
\left|\tilde{e}_{n k}\right|_{p} \leq C n^{2 p} \delta_{k}^{-p}
$$

To deal with $|Q(F)|_{p}$, we use the following decomposition

$$
|Q(F)|_{p} \leq\left(\sum_{k=1}^{4 q} \sum_{n=0}^{q}+\sum_{k=1}^{4 q} \sum_{n=q+1}^{\infty}+\sum_{k=4 q+1}^{\infty} \sum_{n=0}^{l(k)-1}+\sum_{k=4 q+1}^{\infty} \sum_{n=l(k)}^{\infty}\right)\left|\eta_{n k}(f)\right| \cdot\left|\tilde{e}_{n k}\right|_{p} .
$$

Let us consider the sums above separately. We omit the subscripts of $\eta_{n k}$ and $\tilde{e}_{n k}$.

For the first $\operatorname{sum}|\eta(f)| \cdot|\tilde{e}|_{p} \leq C\|f\|_{q} \delta_{4 q}^{-2 p-1} \leq C\|f\|_{q}$, as $k \leq 4 q$.

For the second sum (3.2) and (3.8) imply

$$
|\eta(f)| \cdot|\tilde{e}|_{p} \leq C\left(\delta_{k} / n\right)^{q} n^{2 p} \delta_{k}^{-p}\|f\|_{q},
$$

which is a term of convergent series due to the choice of $q$.

If now $k>4 q$ and $n<l(k)$, then by (3.4) and (3.7)

$$
|\eta(f)| \cdot|\tilde{e}|_{p} \leq C \delta_{k}^{q-1} \delta_{k}^{-2 p-1}\|f\|_{q} .
$$

After summation over $n$ we obtain $l(k)$ terms of this type. Since $l(k)<\delta_{k}^{-1}$, the corresponding series over $k$ is convergent.

In the same manner it can be checked that the last double series converges as well.

Thus the operator $Q$ is well defined and $|Q(F)|_{p} \leq C\|f\|_{q}$. Using the Lagrangian form of the Taylor remainders we have the bound

$$
\left|\left(R_{y}^{q} f\right)^{(i)}(x)\right|=\left|\left(R_{y}^{q} \tilde{f}\right)^{(i)}(x)\right| \leq 2|\tilde{f}|_{q}|x-y|^{q-i}
$$

for any extension $\tilde{f} \in C^{\infty}[0,1]$ of $f$ and $x, y \in K, i \leq q$. Therefore, $\|f\|_{q} \leq 3|\tilde{f}|_{q}$ for any extension $\tilde{f}$ and in particular for $\tilde{f}=F$. This gives the boundedness of the operator $Q$ and proves the lemma. 
Let $X_{1}$ denote $Q\left(C^{\infty}[0,1]\right), X_{0}=\left\{F \in C^{\infty}[0,1]: \operatorname{supp} F \subset \bigcup_{k=2}^{\infty}\left[b_{k}, a_{k-1}\right]\right\}$. Then $C^{\infty}[0,1]=X_{1} \oplus X_{0}$ and $\left(\tilde{e}_{n k}\right)_{n=0, k=1}^{\infty, \infty}$ is a basis in the space $X_{1}$. Let $P_{k}$ denote the following projection: $P_{k}(F)=F-Q(F)$ on $\left[b_{k}, a_{k-1}\right]$ and $P_{k}(F)=0$ otherwise on [0,1]. Clearly, $P_{k}\left(C^{\infty}[0,1]\right) \simeq C_{0}^{\infty}\left[b_{k}, a_{k-1}\right]$, where the isomorphism is just identification of functions from $C_{0}^{\infty}\left[b_{k}, a_{k-1}\right]$ with their extensions on $[0,1]$ by zero. Here and subsequently, $C_{0}^{\infty}[a, b]$ denotes the space of $C^{\infty}$-functions vanishing at the endpoints of the interval $[a, b]$.

Consider the Hermite functions

$$
h_{n}(t)=(-1)^{n}\left(2^{n} n !\right)^{-1 / 2} \pi^{-1 / 4} e^{t^{2} / 2}\left(e^{-t^{2}}\right)^{(n)}, t \in \mathbb{R}, n \in \mathbb{N}_{0} .
$$

They form a basis in the space $\mathcal{S}$ of rapidly decreasing on the line functions ([12], L. 27). The operator $f(t) \mapsto g(x)=f\left(\tan \left(\frac{\pi}{2} x\right)\right)$ gives an isomorphism of the spaces $\mathcal{S}$ and $C_{0}^{\infty}[-1,1]$ ([12], L. 26). Therefore the sequence $\left(\tilde{h}_{n k}\right)_{n=0}^{\infty}$, where $\tilde{h}_{n k}(x)=h_{n}\left(\tan \left(\frac{\pi}{2} \frac{2 x-b_{k}-a_{k-1}}{b_{k}-a_{k-1}}\right)\right)$ for $b_{k}<x<a_{k-1}$ and $\tilde{h}_{n k}(x)=0$ otherwise on $[0,1]$, is a basis in the space $P_{k}\left(C^{\infty}[0,1]\right)$.

The function $F-Q(F)$ is flat on the compact set $K$. Using the Taylor expansion of $P_{k}(F)$ at $a_{k-1}$ it is easy to see that for any $q \in \mathbb{N}_{0}$ the sequence $\left(\left|P_{k}(F)\right|_{q}\right)_{k=2}^{\infty}$ is rapidly decreasing. Therefore,

$$
X_{0}=\left(\oplus_{k=2}^{\infty} P_{k}\left(C^{\infty}[0,1]\right)\right)_{s}
$$

and what is more, for any $p \in \mathbb{N}$ there exists $q \in \mathbb{N}$ and a constant $C$ such that

$$
\left\|\tilde{h}_{n k}\right\|_{p} \cdot\left|\zeta_{n k}\right|_{-q} \leq C, \quad \forall n, k .
$$

Here the system of functionals $\left\{\zeta_{n k}\right\}$ is biorthogonal to $\left\{\tilde{h}_{n k}\right\}$ in the space $C^{\infty}[0,1]$.

Taking into account Lemma 3.1 we see that the system of elements $\tilde{e}_{n k}, \tilde{h}_{n k}$ with the corresponding functionals $\eta_{n k}, \zeta_{n k}$ satisfies the Dynin-Mityagin criterion ([12], T. 9, see also [11]). Thus we have proved the following.

Theorem 3.2. The functions $\tilde{e}_{n k}, \tilde{h}_{n, k+1}, n \in \mathbb{N}_{0}, k \in \mathbb{N}$ form a basis in the space $C^{\infty}[0,1]$.

\section{Graduated sharp cusp}

Let us fix a sequence $\left(\psi_{k}\right)_{k=1}^{\infty}, \psi_{k} \downarrow 0$ and consider the step-function $\psi: \psi(x)=\psi_{k}$ if $a_{k} \leq x<a_{k-1}, k \in \mathbb{N}$. Here we use notations of Section $3, a_{0}=b_{1}=1$.

Let us consider the following domain

$$
\Omega_{\psi}=\left\{(x, y) \in \mathbb{R}^{2}: 0<x<1,|y|<\psi(x)\right\} .
$$

The spaces $C^{\infty}\left(\bar{\Omega}_{\psi}\right)$ and $s$ are isomorphic if and only if there exists a constant $M$ such that $\psi_{k} \geq \delta_{k}^{M}$ for all $k$ ([4], T. 1.3). Moreover, by choosing the suitable scale of sequences $\left(\psi_{k}^{(\alpha)}\right)_{k=1}^{\infty}$ one can get a family having cardinality of the continuum of pair-wise nonisomorphic spaces of this type (see $[14,5,4])$.

We will construct a basis in the space $C^{\infty}\left(\bar{\Omega}_{\psi}\right)$ for the sharp cusp; we can assume that

$$
\psi_{k} \leq \delta_{k}^{2}, \quad k \in \mathbb{N}
$$

Let us denote by $R_{k}$ the rectangle $I_{k} \times\left[-\psi_{k}, \psi_{k}\right]$, by $R_{k}^{\prime}$ the rectangle $\left[b_{k}, a_{k-1}\right] \times\left[-\psi_{k}, \psi_{k}\right]$. Let $K=\{0\} \cup \bigcup_{k=1}^{\infty} R_{k}$. 
At first we give a basis in the space $\mathcal{E}(K)$.

Let $e_{n m k}(x, y)=\left.e_{n k}(x) T_{m}\left(\frac{y}{\psi_{k}}\right)\right|_{K}, n, m \in \mathbb{N}_{0}, k \in \mathbb{N}$. For $f \in \mathcal{E}(K)$ let

$$
\xi_{n m k}(f)=\frac{4}{\pi^{2}} \int_{0}^{\pi} \int_{0}^{\pi} f\left(x_{k}+\delta_{k} \cos t, \psi_{k} \cos \tau\right) \cos n t \cos m \tau d t d \tau
$$

(here and in what follows instead of 4 we take 1 if $n=m=0$ or 2 if $n m=0, n+m \neq 0$ ). Set $\eta_{n m k}(f)=\xi_{n m k}(f)$ for $n \geq l(k)=[k / 4]$. If $n<l(k)$ then let

$$
\begin{aligned}
\eta_{n m k}(f)= & \frac{4}{\pi^{2}} \int_{0}^{\pi} \int_{0}^{\pi}\left[f\left(x_{k}+\delta_{k} \cos t, \psi_{k} \cos \tau\right) \cos n t\right. \\
& \left.-f\left(x_{k-1}+\delta_{k-1} \cos t, \psi_{k} \cos \tau\right) \cdot \sum_{i=n}^{l-1} \xi(e) \cos i t\right] \cos m \tau d t d \tau .
\end{aligned}
$$

Here as in [7] we use the notation

$$
\sum_{i=n}^{l-1} \xi(e):=\sum_{i=n}^{l(k-1)-1} \xi_{n k}\left(e_{i k-1}\right) .
$$

Lemma 4.1. The system of functionals $\left(\eta_{n m k}\right)_{n, m=0, k=1}^{\infty, \infty}$ is total on $\mathcal{E}(K)$ and biorthogonal to $\left(e_{n m k}\right)_{n, m=0, k=1}^{\infty, \infty}$.

Proof. Let $\xi_{m k}^{(Y)}$ denote the functional $\xi_{m k}^{(Y)}(G)=\frac{2}{\pi} \int_{0}^{\pi} G\left(\psi_{k} \cos \tau\right) \cos m \tau d \tau$. If the function $f \in \mathcal{E}(K)$ can be represented in the form $f(x, y)=F(x) G(y)$, then, as is easy to see, $\eta_{n m k}(f)=\eta_{n k}(F) \xi_{m k}^{(Y)}(G)$. This remark and biorthogonality of the system $\left\{e_{n k}, \eta_{n k}\right\}$ imply that of $\left\{e_{n m k}, \eta_{n m k}\right\}$. The property of being total can be proved in the same manner as in Lemma 3.2 in [7].

Theorem 4.2. The system $\left\{e_{n m k}, \eta_{n m k}\right\}_{n, m=0, k=1}^{\infty, \infty}$ is a basis in the space $\mathcal{E}(K)$.

Proof. According to the Dynin-Mityagin criterion and Lemma 4.1 it is enough to show that for any $p \in \mathbb{N}$ there exists $q \in \mathbb{N}$ and a constant $C$ such that

$$
\left\|e_{n m k}\right\|_{p} \cdot\left|\eta_{n m k}\right|_{-q} \leq C, \quad \forall n, m, k .
$$

For given $p$ let us fix $q=2 p+2, k_{q}=4 q+3$ and natural $m_{q} \geq q \delta_{k_{q}}^{-1 / 2}$. We decompose $\mathbb{N}_{0}^{2} \times \mathbb{N}$ into certain zones and give the estimations of the norms for given cases separately. To simplify notations we use the same letter $C$ for any constant which does not depend on $n, m$ and $k$. The details are left to the reader.

Z0. $n \leq q, m \leq m_{q}, k \leq k_{q}$. Here we have only finite number of elements and no problem with (4.2).

$$
\begin{aligned}
& \text { If } n \geq l(k) \text {, then } e_{n m k}(x, y)=T_{n}\left(\frac{x-x_{k}}{\delta_{k}}\right) T_{m}\left(\frac{y}{\psi_{k}}\right) \text { for }(x, y) \in R_{k} . \\
& \text { If } j=\left(j_{1}, j_{2}\right) \in \mathbb{N}_{0}^{2}, \quad j_{1} \leq n, j_{2} \leq m \text {, then }
\end{aligned}
$$

$$
\left|e_{n m k}^{(j)}\right| \leq\left(\frac{n^{2}}{\delta_{k}}\right)^{j_{1}}\left(\frac{m^{2}}{\psi_{k}}\right)^{j_{2}} .
$$


Here we have to take into account what term in the product above is larger and will separate the cases when the inequality

$$
\frac{n^{2}}{\delta_{k}}>\frac{m^{2}}{\psi_{k}}
$$

holds.

Z1.1. $n \geq l(k)$ and (4.3) is valid (except the points from the zone Z0).

Here $\left\|e_{n m k}\right\|_{p} \leq C\left(\frac{n^{2}}{\delta_{k}}\right)^{p}$.

Z1.2. $n \geq l(k),(4.3)$ is not valid and $m>p \Longrightarrow\left\|e_{n m k}\right\|_{p} \leq C\left(\frac{m^{2}}{\psi_{k}}\right)^{p}$.

Z1.3. $n \geq l(k),(4.3)$ is not valid and $m \leq p \Longrightarrow\left\|e_{n m k}\right\|_{p} \leq C \psi_{k}^{-m}\left(\frac{n^{2}}{\delta_{k}}\right)^{p-m}$.

Consider now the cases with $n<l(k)$. Here

$$
e:=e_{n m k}(x, y)=\tilde{T}_{n k}(x) T_{m}\left(\frac{y}{\psi_{k}}\right)
$$

for $(x, y) \in \bigcup_{q=k}^{\infty} R_{q}$ and it is 0 otherwise on $K$. We conclude from (3.3) and (4.1) that

$$
\left|e^{(j)}\right| \leq \delta_{k}^{-1-2 j_{1}}\left(\frac{m^{2}}{\psi_{k}}\right)^{j_{2}} \leq \delta_{k}^{-1} \psi_{k}^{-|j|} m^{2 j_{2}}
$$

if $j_{1} \leq n, j_{2} \leq m$.

Z2.1. $n<l(k), p<m \Longrightarrow\left\|e_{n m k}\right\|_{p} \leq C \delta_{k}^{-1}\left(\frac{m^{2}}{\psi_{k}}\right)^{p}$.

Z2.2. $n<l(k), m \leq p<m+n \Longrightarrow\left\|e_{n m k}\right\|_{p} \leq C \delta_{k}^{-1-2(p-m)}\left(\frac{m^{2}}{\psi_{k}}\right)^{m} \leq C \psi_{k}^{-m} \delta_{k}^{-1-2(p-m)}$.

Z2.3. $n<l(k), m+n \leq p \Longrightarrow\left\|e_{n m k}\right\|_{p} \leq C \psi_{k}^{-m} \delta_{k}^{m-n-p-1}$.

Our next objective is to evaluate the dual norms of the functionals $\eta_{n m k}$.

Let the function $g$ be $r$ times differentiable on the interval $I$ with length $\Delta$. Then for the best approximation to $g$ by polynomials of degree $\leq n$ on $I$ in the norm $|\cdot|_{0}$ we have the following form of the Jackson theorem (see e. g., [15], 5.1.5):

$$
E_{n}(g, I) \leq C_{r}\left(\frac{\Delta}{n}\right)^{r}\left|g^{(r)}\right|_{0}, n \geq r,
$$

where the constant $C_{r}$ does not depend on $n, g$ and $\Delta$.

Let us consider now all given zones in the same order. Fix $f \in \mathcal{E}(K)$.

$Z^{\prime} 1.1 . n \geq l(k)$ with (4.3). Here

$$
\eta_{n m k}(f)=\xi_{n m k}(f)=\frac{4}{\pi^{2}} \int_{0}^{\pi}\left[\int_{0}^{\pi} f\left(x_{k}+\delta_{k} \cos t, \psi_{k} \cos \tau\right) \cos n t d t\right] \cos m \tau d \tau .
$$

Due to orthogonality we can subtract from $f$ in the internal integral arbitrary polynomial $Q_{n-1}$ $(\cos t)$ of degree $\leq n-1$. If we take the polynomial of the best approximation to $f$ with respect 
to the first variable then we get by (4.4)

$$
\left|\eta_{n m k}(f)\right| \leq \frac{4}{\pi} \int_{0}^{\pi} C_{q}\left(\frac{\delta_{k}}{n-1}\right)^{q}|f|_{q} d \tau \leq C\left(\frac{\delta_{k}}{n}\right)^{q}\|f\|_{q},
$$

as $n-1 \geq q$.

$Z^{\prime} 1.2 . n \geq l(k),(4.3)$ is not valid, $m>p$. We apply the previous argument with the polynomial $Q_{m-1}$ of the best approximation to $f$ with respect to the second variable.

If $m>q$, then $\left|\eta_{n m k}(f)\right| \leq C\left(\frac{\psi_{k}}{m}\right)^{q}\|f\|_{q}$.

If $p<m \leq q$, then

$$
\left|\eta_{n m k}(f)\right| \leq C\left(\frac{\psi_{k}}{m-1}\right)^{m-1}\|f\|_{m-1} \leq C \psi_{k}^{p}\|f\|_{q}
$$

and that is enough for (4.2) since in the bound of $\left\|e_{n m k}\right\|_{p}$ for this case we can replace $m^{2 p}$ by a constant.

$Z^{\prime} 1.3 . n \geq l(k),(4.3)$ is not valid, $m \leq p$. Here at first we take the Taylor expansion of $f$ with respect to the second variable:

$$
\xi_{n m k}(f)=\frac{4}{\pi^{2}} \int_{0}^{\pi} \int_{0}^{\pi} f_{y^{m}}^{(m)}\left(x_{k}+\delta_{k} \cos t, \theta\right) \cos n t \frac{\psi_{k}^{m}}{m !} \cos ^{m} \tau \cos m \tau d t d \tau,
$$

$\theta \in\left[-\psi_{k}, \psi_{k}\right]$

Since $n-1 \geq q$, we can apply (4.4) with $r=q-m$ to $f_{y^{m}}^{(m)}(\cdot, \theta)$ :

$$
\left|\eta_{n m k}(f)\right| \leq C \psi_{k}^{m}\left(\frac{\delta_{k}}{n-1}\right)^{q-m}\left|f_{x^{q-m} y^{m}}^{(q)}\right|_{0} \leq C \psi_{k}^{m}\left(\frac{\delta_{k}}{n}\right)^{q-m}\|f\|_{q} .
$$

We now turn to the cases when $n<l(k)$. Here the functional $\eta_{n m k}$ is represented by

$$
\begin{aligned}
\eta_{n m k}(f)= & \frac{4}{\pi^{2}} \int_{0}^{\pi} \int_{0}^{\pi}\left[f\left(x_{k}+\delta_{k} \cos t, \psi_{k} \cos \tau\right) \cos n t\right. \\
& \left.-f\left(x_{k-1}+\delta_{k-1} \cos t, \psi_{k} \cos \tau\right) \cdot \sum_{i=n}^{l-1} \xi(e) \cos i t\right] \cos m \tau d t d \tau
\end{aligned}
$$

We will use the bound (6.1) from [7]:

$$
\sum_{i=n}^{l(k-1)-1}\left|\xi_{n k}\left(e_{i k-1}\right)\right|<\delta_{k}^{-1}
$$

$Z^{\prime} 2.1 . n<l(k), m>p$. If $m>q$, then we subtract from both functions above the polynomials $Q_{m-1}, \tilde{Q}_{m-1}$ of the best approximation to the corresponding functions with respect to the second variable. In both cases we get the bound $|f-Q|_{0} \leq C_{q}\left(\frac{\psi_{k}}{m}\right)^{q}|f|_{q}$, as $m-1 \geq q$. Therefore,

$$
\left|\eta_{n m k}\right|_{-q} \leq C \delta_{k}^{-1}\left(\frac{\psi_{k}}{m}\right)^{q}
$$


If $p<m \leq q$, then $\left|\eta_{n m k}(f)\right| \leq C \psi_{k}^{m} \delta_{k}^{-1}|f|_{m}$ and

$$
\left\|e_{n m k}\right\|_{p}\left|\eta_{n m k}\right|_{-q} \leq C \psi_{k}^{m-p} \delta_{k}^{-2} \leq C,
$$

by $(4.1)$.

$Z^{\prime}$ 2.2. $n<l(k), m \leq p<m+n$. First suppose that $m+n \geq q$. Then we use the Taylor expansion of both functions with respect to $y$ at 0 up to $m$-th degree and then expand the first function at $x_{k}$, the second term at $x_{k-1}$ up to $(q-m)$-th degree. Since $n \geq q-m$ and $i \geq n$ all terms of the small degree will vanish after integration. From this we deduce that

$$
\left|\eta_{n m k}\right|_{-q} \leq C \psi_{k}^{m}\left[\delta_{k}^{q-m}+\delta_{k-1}^{q-m} \delta_{k}^{-1}\right] \leq C \psi_{k}^{m} \delta_{k}^{q-m-1},
$$

as $\delta_{k-1}=2 \delta_{k}$.

Now we have the most difficult case: $m \leq p<m+n<q$. Take the Taylor expansion of the functions with respect to $y$ at 0 up to $(q-n-1)$-th degree. Then

$$
\begin{aligned}
& \eta_{n m k}(f)=\frac{4}{\pi^{2}} \int_{0}^{\pi} \int_{0}^{\pi}\left[\sum_{s=m}^{q-n-1} f_{y^{s}}^{(s)}\left(x_{k}+\delta_{k} \cos t, 0\right) \frac{\psi_{k}^{s}}{s !} \cos ^{s} \tau \cos n t\right. \\
& \left.-\sum_{s=m}^{q-n-1} f_{y^{s}}^{(s)}\left(x_{k-1}+\delta_{k-1} \cos t, 0\right) \frac{\psi_{k}^{s}}{s !} \cos ^{s} \tau \sum_{i=n}^{l-1} \xi(e) \cos i t\right] \cos m \tau d t d \tau+\text { Remainder },
\end{aligned}
$$

where

$$
\begin{aligned}
& \text { Remainder }=\frac{4}{\pi^{2}} \int_{0}^{\pi} \int_{0}^{\pi}\left[f_{y^{q-n}}^{(q-n)}\left(x_{k}+\delta_{k} \cos t, \theta_{1}\right) \frac{\psi_{k}^{q-n}}{(q-n) !} \cos ^{q-n} \tau \cos n t\right. \\
& \left.-f_{y^{q-n}}^{(q-n)}\left(x_{k-1}+\delta_{k-1} \cos t, \theta_{2}\right) \frac{\psi_{k}^{q-n}}{(q-n) !} \cos ^{q-n} \tau \sum_{i=n}^{l-1} \xi(e) \cos i t\right] \cos m \tau d t d \tau,
\end{aligned}
$$

$\theta_{1}, \theta_{2} \in\left[-\psi_{k}, \psi_{k}\right]$. The terms corresponding to values $s=0,1, \ldots, m-1$ vanish after integration. In Remainder we take the expansions of $f$ with respect to $x$ up to $n$-th degree at $x_{k}$ and $x_{k-1}$ correspondingly. Then

$$
\mid \text { Remainder }\left.\left|\leq C \psi_{k}^{q-n}\left[\delta_{k}^{n}+\delta_{k-1}^{n} \delta_{k}^{-1}\right]\right| f\right|_{q} \leq C \psi_{k}^{q-n} \delta_{k}^{n-1}|f|_{q} .
$$

By (4.1), the product of this value with $\left\|e_{n m k}\right\|_{p}$ is uniformly bounded, as is easy to check, thus we can turn to the main part of the expansion of $\xi_{n m k}(f)$.

Let $g$ denote the function $f_{y^{s}}^{(s)}(\cdot, 0)$. Then we get the following representation of Main part:

$$
\begin{aligned}
& \sum_{s=m}^{q-n-1} \frac{2}{\pi} \frac{\psi_{k}^{s}}{s !} \int_{0}^{\pi} \cos ^{s} \tau \cos m \tau\left\{2 / \pi \int_{0}^{\pi}\left[g\left(x_{k}+\delta_{k} \cos t\right) \cos n t\right.\right. \\
& \left.\left.-g\left(x_{k-1}+\delta_{k-1} \cos t\right) \sum_{i=n}^{l-1} \xi(e) \cos i t\right] d t\right\} d \tau .
\end{aligned}
$$

The expression in the braces is $\eta_{n k}(g)$. By Lemma 4.1 in [7]

$$
\left|\eta_{n k}(g)\right| \leq C\left[\delta_{k}^{q-s}+\left(b_{k-1}-x_{k}\right)^{q-s} \delta_{k}^{-1}\right]\|g\|_{q-s}
$$


as $n<q-s$. This implies

$$
\left|\eta_{n k}(g)\right| \leq C \delta_{k}^{q-s-1}\|f\|_{q}
$$

and we get the bound

$$
\mid \text { Main part } \mid \leq C \sum_{s=m}^{q-n-1} \psi_{k}^{s} \delta_{k}^{q-s-1}\|f\|_{q} .
$$

Clearly that the maximal term in the sum corresponds to $s=m$. Thus,

$$
\mid \text { Main part } \mid \leq C \psi_{k}^{m} \delta_{k}^{q-m-1}\|f\|_{q},
$$

which is enough to neutralize the value $\psi_{k}^{-m} \delta_{k}^{-1-2 p+2 m}$ in $\left\|e_{n m k}\right\|_{p}$ due to the choice of $q$.

$Z^{\prime} 2.3 . n<l(k), m+n \leq p$. All arguments of the case $p<m+n<q$ can be repeated.

Thus for the system $\left\{e_{n m k}, \eta_{n m k}\right\}_{n, m=0, k=1}^{\infty, \infty}$ we have the Dynin-Mityagin condition (4.2), and the proof is complete.

We are able now to construct a basis in the space $C^{\infty}\left(\bar{\Omega}_{\psi}\right)$. The result follows by the same method as in the construction of the special basis in the space $C^{\infty}[0,1]$. Using the same notations as in Section 3 we introduce

$$
\tilde{e}_{n m k}(x, y)=\tilde{e}_{n k}(x) T_{m}\left(\frac{y}{\psi_{k}}\right),(x, y) \in \Omega_{\psi} .
$$

For given $F \in C^{\infty}\left(\bar{\Omega}_{\psi}\right)$ we consider the restriction $f$ of $F$ on $K$ as an element of the space $\mathcal{E}(K)$. The derivative $\tilde{e}_{n k}^{\left(j_{1}\right)}(x)$ has the same (up to a constant) upper bound as $e_{n k}^{\left(j_{1}\right)}(x)$ due to the choice of the smoothing functions $\omega_{n k}$. Therefore the projection

$$
Q: C^{\infty}\left(\bar{\Omega}_{\psi}\right) \rightarrow C^{\infty}\left(\bar{\Omega}_{\psi}\right): F \mapsto \sum_{k=1}^{\infty} \sum_{n=0}^{\infty} \sum_{m=0}^{\infty} \eta_{n m k}(f) \cdot \tilde{e}_{n m k}
$$

is well defined and continuous.

Now let $Y_{1}=Q\left(C^{\infty}\left(\bar{\Omega}_{\psi}\right)\right), Y_{0}=\left\{F \in C^{\infty}\left(\bar{\Omega}_{\psi}\right): \operatorname{supp} F \subset \bigcup_{k=2}^{\infty} R_{k}^{\prime}\right\}$. Then $C^{\infty}\left(\bar{\Omega}_{\psi}\right)=Y_{1} \oplus Y_{0}$ and $\left(\tilde{e}_{n m k}\right)_{n, m=0, k=1}^{\infty, \infty}$ is a basis in the space $Y_{1}$. Take the projection: $P_{k}(F)=F-Q(F)$ on $R_{k}^{\prime}$ and 0 otherwise on $\bar{\Omega}_{\psi}$. Here $P_{k}\left(C^{\infty}\left(\bar{\Omega}_{\psi}\right)\right)=\left\{F \in C^{\infty}\left(\bar{\Omega}_{\psi}\right)\right.$ : $\left.\operatorname{supp} F \subset R_{k}^{\prime}\right\}=C_{0}^{\infty}\left[b_{k}, a_{k-1}\right] \tilde{\otimes} C^{\infty}\left[-\psi_{k}, \psi_{k}\right]$. Arguing as in Section 3, we can see that the functions $\left(\tilde{h}_{n m k}\right)_{n, m=0}^{\infty}$, where $\tilde{h}_{n m k}(x, y)=\tilde{h}_{n k}(x) T_{m}\left(\frac{y}{\psi_{k}}\right)$, form the basis in the subspace $P_{k}\left(C^{\infty}\left(\bar{\Omega}_{\psi}\right)\right)$ of the space $Y_{0}$ and

$$
Y_{0}=\left(\oplus_{k=2}^{\infty} P_{k}\left(C^{\infty}\left(\bar{\Omega}_{\psi}\right)\right)\right)_{s} .
$$

Arguing as in the proof of Theorem 3.2, we have the following theorem.

Theorem 4.3. The functions $\tilde{e}_{n m k}, \tilde{h}_{n, m, k+1}, n, m \in \mathbb{N}_{0}, k \in \mathbb{N}$ form a basis in the space $C^{\infty}\left(\bar{\Omega}_{\psi}\right)$.

Let us note that the space $X_{0}$ in Theorem 3.2 is isomorphic to $s$ (see e. g., [11], Proposition 31.12). On the other hand, for the present case we have the following. 
Proposition 4.4. The spaces $Y_{0}$ and $s$ are isomorphic if and only if for some constant $M$

$$
\psi_{k} \geq \delta_{k}^{M}, k \in \mathbb{N}
$$

Proof. Suppose that $Y_{0} \simeq s$ and therefore the space $Y_{0}$ belongs to the class $D_{1}[18]$ or has the dominating norm property DN (see e. g., [11]), that is $\exists p: \forall q \exists r, C$

$$
|f|_{q}^{2} \leq C|f|_{p}|f|_{r}, \quad f \in X_{0}
$$

where $p, q, r \in \mathbb{N}_{0}, C>0$.

Suppose, contrary to our claim that there exists a subsequence $\left(k_{l}\right)_{l=1}^{\infty}$ with

$$
\psi_{k_{l}}<\delta_{k_{l}}^{l}, l \in \mathbb{N}
$$

Let $p \in \mathbb{N}_{0}$ be chosen from the definition of the class $D_{1}$. For $q=p+1$ let $r$ and $C$ be fixed from the definition (4.6). Let us consider the functions $f_{l}, l \in \mathbb{N}$ with $f_{l}(x, y)=y^{q} \omega_{0 k}(x)$. Then, as is easy to check, $\left|f_{l}\right|_{p} \leq C \psi_{k_{l}} \delta_{k_{l}}^{-p},\left|f_{l}\right|_{r} \leq C \delta_{k_{l}}^{-r+q}$ and $\left|f_{l}\right|_{q} \geq\left|\left(f_{l}\right)_{y^{q}}^{(q)}(0,0)\right| \geq 1$. Using the supposition about $\psi_{k_{l}}$ we obtain

$$
1 \leq\left|f_{l}\right|_{q}^{2} \leq C\left|f_{l}\right|_{p}\left|f_{l}\right|_{r} \leq C \delta_{k_{l}}^{l+q-r-p},
$$

which is a contradiction for large $l$.

For the inverse implication we see that (4.5) implies the isomorphism $C^{\infty}\left(\bar{\Omega}_{\psi}\right) \simeq s$. Therefore the space $Y_{0}$ can be considered as a complemented subspace of $s$. On the other hand, $Y_{0}$ contains a complemented subspace which is isomorphic to $s$ (for example, the subspace $P_{k}\left(C^{\infty}\left(\bar{\Omega}_{\psi}\right)\right)$ for any fixed $\left.k\right)$. Using the Pelczyński-Vogt decomposition method (see e. g., [11], L. 31.2), we get the desired conclusion.

\section{References}

[1] Baouendi, M.S. and Goulaouic, C. Régularité analytique et itérés d'opérateurs elliptiques dégénérés; application, J. Funct. Anal., 9, 208-248, (1972).

[2] Djakov, P.B. and Mityagin, B.S. Modified construction of a nuclear Fréchet space without basis, J. Funct. Anal, 23, 415-433, (1976).

[3] Domański, P. and Vogt, D. The space of real-analytic functions has no basis, Studia Math, 142(2), 187-200, (2000)

[4] Goncharov, A.P. Isomorphic classification of the spaces of infinitely differentiable functions, Ph.D. Thesis, Rostov State University, (1986), (Russian).

[5] Goncharov, A.P. and Zahariuta, V.P. Linear topological invariants and spaces of infinitely differentiable functions, Math. Analiz. i ego priloz., Rostov State University, 18-27, (1985), (Russian).

[6] Goncharov, A.P. and Zahariuta, V.P. On the existence of basis in spaces of Whitney functions on special compact sets in $\mathbb{R}$, METU, preprint Series 93/58, Ankara, Turkey.

[7] Goncharov, A.P. Spaces of Whitney functions with basis, Math. Nachr., 220, 45-57, (2000).

[8] Guillemot-Teissier, M. Séries de Legendre des distributions: Structures hilbertiennes, C.R. Acad. Sci. Paris, 265, A461-A464, (1967).

[9] Kondakov, V.P. and Zahariuta, V.P. On bases in spaces of infinitely differentiable functions on special domains with cusp, Note di Matematica, XII, 99-106, (1992).

[10] Malgrange, B. Ideals of Differentiable Functions, Oxford University Press, 1966.

[11] Meise, R. and Vogt, D. Introduction to Functional Analysis, Oxford, Clarendon Press, 1997

[12] Mityagin, B.S. Approximate dimension and bases in nuclear spaces, Russian Math. Surveys, 16(4), 59-127, (1961).

[13] Tidten, M. Fortsetzungen von $C^{\infty}$-Funktionen, welche auf einer abgeschlossenen Menge in $\mathbb{R}^{n}$ definiert sind, Manuscripta Math., 27, 291-312, (1979). 
[14] Tidten, M. An example of continuum of pairwise non-isomorphic spaces of $C^{\infty}$-functions, Studia Math., 78, 267-274, (1984).

[15] Timan, A.F. Theory of Approximation of Functions of a Real Variable, Pergamon Press, 1963.

[16] Triebel, H. Erzeugung des nuklearen lokalkonvexen Räumes $C^{\infty}(\bar{\Omega})$ durch einen elliptischen Differentialoperator zweither Ordnung, Math. Ann., 177, 247-264, (1968).

[17] Vogt, D. Sequence space representations of spaces of test functions and distributions, in Functional Analysis, Holomorphy and Approximation Theory, Zapata, G.I., Ed., Lecture Notes in Pure and Appl. Math., 83, Dekker, 405-443, (1983).

[18] Zahariuta, V.P. Some linear topological invariants and isomorphisms of tensor products of scale's centers, $I z v$. Sev. Kavkaz. Nauch. Centra Vysh. Skoly., 4, 405-443, (1974), (Russian).

[19] Zeriahi, A. Inégalités de Markov et Développement en Série de Polynômes Orthogonaux des Fonctions $C^{\infty}$ et $A^{\infty}$, Several Complex Variables: Proceedings of the Mittag-Leffler Institute, (1987-1988), Fornaess, J.F., Ed., Math. Notes, 38, Princeton University Press, Princeton, New Jersey, 684-701, 1993.

[20] Zerner, M. Développement en séries de polynômes orthonormaux des fonctions indéfiniment différentiables, C.R. Acad. Sci. Paris, 268, 218-220, (1969).

[21] Zobin, N.M. and Mityagin, B.S. Examples of nuclear Fréchet spaces without basis, Funct. Anal. i ego priloz., 84, 35-47, (1974), (Russian).

Received August 13, 2001

Revision received May 17, 2002

Bilkent University, 06533 Ankara, Turkey

and

Civil Building University, Rostov-on-Don, Russia e-mail: goncha@fen.bilkent.edu.tr

Rostov State University, Rostov-on-Don, Russia and

Middle East Technical University, Ankara, Turkey e-mail: zaha@math.metu.edu.tr 